\title{
High energy secondary peak structure in tunneling spectra (hump) as possible magnetic pseudogap
}

\author{
J.F. Zasadzinskia,b,*, L. Ozyuzer ${ }^{b, c}$, N. Miyakawa ${ }^{d}$, D.G. Hinks ${ }^{b}$, K.E. Gray ${ }^{b}$

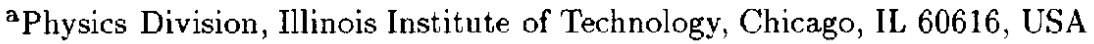 \\ ${ }^{b}$ Materials Science Division, Argonne National Laboratory, Argonne, IL 60439, USA \\ ${ }^{c}$ Department of Physics, Izmir Institute of Technology, TR-35230 Izmir, Turkey \\ ${ }^{d}$ Department of Applied Physics, Science University of Tokyo, Tokyo 162-8601, Japan
}

It is demonstrated that tunneling spectra in various high $T_{c}$ cuprates display generic features. The principal conductance peaks in superconductor-insulator-normal metal (SIN) junctions indicate the superconducting gap in the density of states (DOS). Higher energy features include a dip and hump structure with a strength that is asymmetric in bias voltage. The dip and hump features follow the doping trends of the superconducting gap, $\Delta$, with a rough scaling as $\sim 2 \Delta$ and $\sim 3 \Delta$ respectively. Tunneling spectra in underdoped $\mathrm{Bi}_{2} \mathrm{Sr}_{2} \mathrm{CaCu}_{2} \mathrm{O}_{8+\delta}$ display a more pronounced hump feature suggestive of a second gap in the DOS. It is observed that the hump feature in the tunneling density of states is consistent with other experimental observations of the so-called high energy pseudogap which may have magnetic origins.

\section{INTRODUCTION}

One of the confusing aspects of the pseudogap in high $\mathrm{T}_{c}$ cuprates concerns its energy scale as found in various experiments. Tunneling, ARPES and optical conductivity measurements $[1,2]$ indicate that the pseudogap has an energy scale close to the measured, lowtemperature superconducting gap, $\Delta$, which for optimal doped $\mathrm{Bi}_{2} \mathrm{Sr}_{2} \mathrm{CaCu}_{2} \mathrm{O}_{8+\delta} \quad(\mathrm{Bi2212}$ ) is about $35-40 \mathrm{meV}$. This of course is consistent with scenarios whereby the pseudogap is due to some type of precursor superconductivity [2]. However, other experiments display pseudogap type effects at a much higher energy scale, on the order of the superexchange energy, J. For example, Raman measurements [3] of optimally doped $\mathrm{Bi} 2212$ in the $\mathrm{B}_{2 g}+\mathrm{A}_{1 g}$ symmetry show a suppression of spectral weight below $\mathrm{T}_{c}$ which is strongest around $125 \mathrm{meV}$. Other Raman data show high energy suppression of spectral weight above $\mathrm{T}_{c}$ [4]. In the case of $\mathrm{La}_{1.85} \mathrm{Sr}_{0.15} \mathrm{CuO}_{4}$ angle integrated photoemission (PES) reveal a clear pseudogap above $\mathrm{T}_{c}$ with a size of $30-35 \mathrm{meV}$ which is about three times the superconducting gap for this compound [5]. We suggest here that these observations of higher energy pseudogaps are connected to the higher energy dip and hump features found in quasiparticle tunneling spectra. The observation of asymmetric, high energy dip and hump features in the SIN tunneling spectra of $\mathrm{Bi} 2212$ is well documented [1]. The correlation of these features with similar ones found in SIS junctions has also been established $[1,6]$. The fact that the energies of the dip/hump features follow the trends of the superconducting gap in Bi2212 indicates that they are linked to the interactions responsible for superconductivity. We first show here that these spectral features are not unique to Bi2212 but in fact are found in several other cuprate superconductors.

\footnotetext{
*asadzinski@anl.gov, work supported by US-DOE BESMS, W-31-109-ENG-38 and NSF Office of Science and Technology Centers, DMR 91-20000.
} 


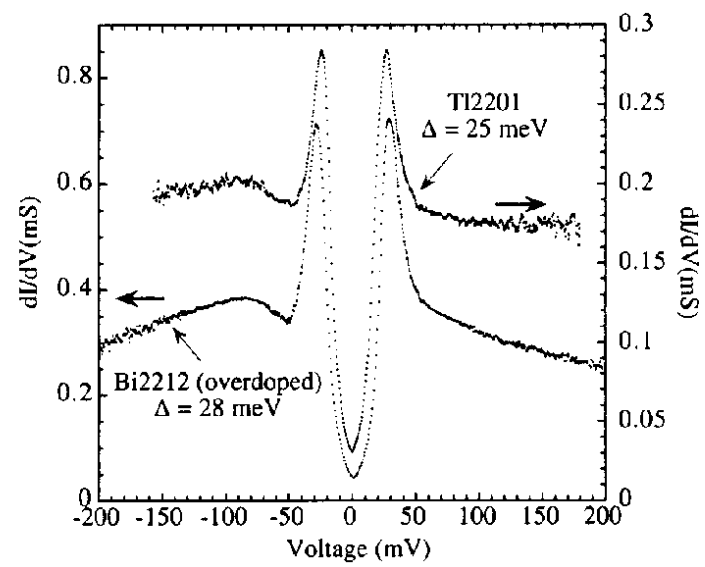

Figure 1. Comparison of SIN conductances of T12201 and slightly overdoped Bi2212.

\section{EXPERIMENTAL RESULTS}

All results presented here have been obtained by point contact tunneling (PCT) using a Au tip [1]. To be sure these are intrinsic properties of the quasiparticles in the $\mathrm{Cu}-\mathrm{O}$ planes it is necessary to establish that they are universal to cuprate superconductors. In Fig. 1 we show the SIN tunneling conductance of $\mathrm{Tl}_{2} \mathrm{Ba}_{2} \mathrm{CuO}_{6}(\mathrm{Tl} 2201)$ obtained by PCT which exhibited a maximum gap value, $\Delta=25 \mathrm{meV}$, for crystals with as-grown $\mathbf{T}_{c}$ $=86 \mathrm{~K}$. For comparison we show the SIN spectrum for a slightly overdoped $\mathrm{Bi} 2212\left(\mathrm{~T}_{c}=82 \mathrm{~K}\right)$ crystal which displays a similar gap value. Both spectra reveal sharp conductance peaks and a cusp feature at, zero bias which are consistent with a d-wave superconducting density of states. For negative bias, which corresponds to electron removal from the superconductor, a pronounced dip feature is observed at $\sim 2 \Delta$, followed by a higher energy hump feature near $80 \mathrm{meV}$ or slightly more than $3 \Delta$. This trend of the hump feature scaling with the superconducting gap exists over the entire doping range [6]. For positive bias there is no sharp dip feature but rather an abrupt change of slope and the hump is barely discernible. This asymmetry is found in most spectra obtained by point contact methods. STM measurements of

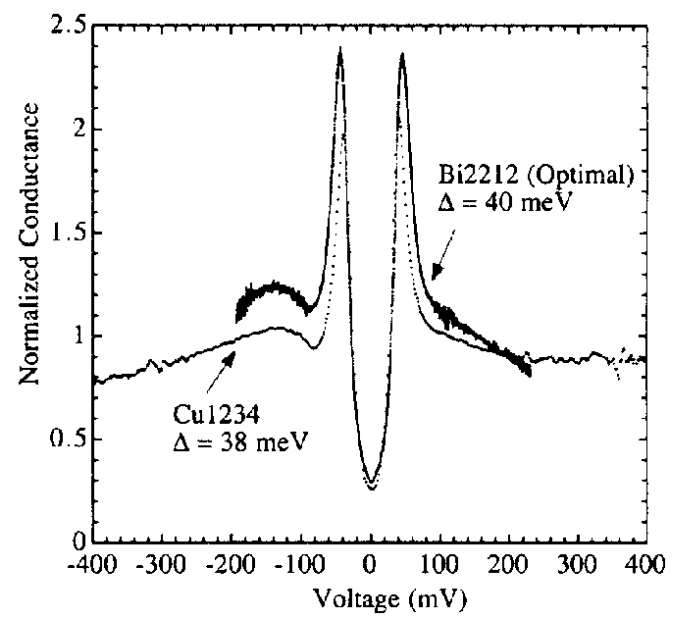

Figure 2. Comparison of SIN conductances of Cu1234 and optimal doped Bi2212.

Bi2212 reveal less asymmetry and the dip/hump is more clearly resolved for positive bias [7]. The origin of this effect is not known but may be related to the different tunneling matrix elements for vacuum and PCT junctions along the c-axis. We note that the clear observations of the cusp feature in PCT junctions indicates that the nodal regions of the Fermi surface are contributing to the tunnel current. But these regions are not expected to produce the dip/hump features as will be discussed below. Overall the SIN spectra of the TI2201 and Bi2212 are very similar indicating no difference between single and double $\mathrm{Cu}-$ O planes per unit cell. This also shows that the dip/hump features are not due to properties of the layers adjacent to the $\mathrm{Cu}-\mathrm{O}$ planes or proximity effects.

To continue the comparison of $\mathrm{Bi2212}$ with other cuprates we show in Fig. 2 the SIN spectrum for a compound $\mathrm{CuBa}_{2} \mathrm{Ca}_{3} \mathrm{Cu}_{4} \mathrm{O}_{y}(\mathrm{Cu} 1234)$ which has a $\mathrm{T}_{c}$ onset of $113 \mathrm{~K}$. The Cu1234 spectrum exhibits a superconducting gap, $\Delta=38$ meV, and is thus compared with a spectrum on optimal doped Bi2212 which shows a similar gap value. Again one observes the sharp conductance peaks, cusp feature at zero bias and asymmetric dip/hump features at nearly the same locations 


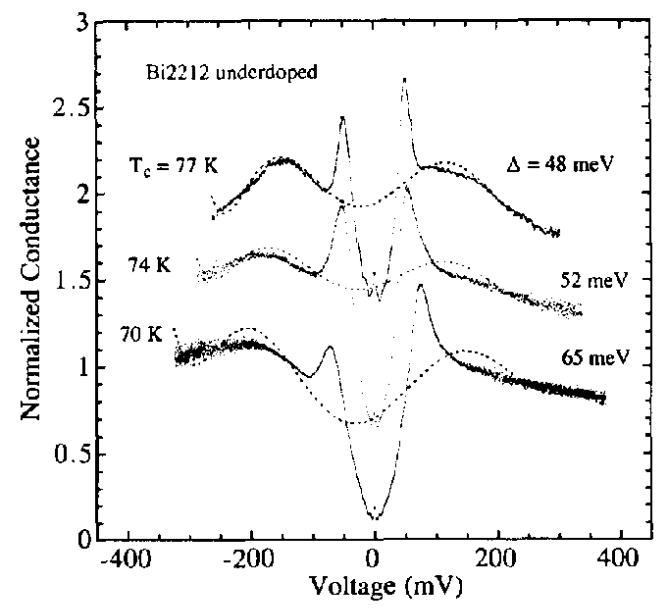

Figure 3. SIN tunneling conductances for three underdoped Bi2212 crystals. Dashed lines are 7 th order polynomial fits of the entire spectrum.

for the two different crystals. Here the hump feature is at approximately $130 \mathrm{meV}$, slightly larger than $3 \Delta$. Note that $\mathrm{Cu} 1234$ has $4 \mathrm{Cu}$ $O$ planes per unit cell. We have thus demonstrated that these spectral features are observed on three very different cuprate superconductors and therefore that they are intrinsic properties of the quasiparticles in the $\mathrm{Cu}-\mathrm{O}$ planes. Furthermore, we have established that the locations of the dip/hump features are tied to the supcrconducting gap value, independent of the the particular cuprate being studied.

Further insight into the dip/hump can be found by examining underdoped $\mathrm{Bi} 2212$ where it is observed that the superconducting gap continues to increase even though $T_{c}$ decreases [1]. In Fig. 3 is shown the SIN spectra from PCT junctions on three underdoped Bi2212 crystals. In each case, the superconducting gap parameter has been obtained by fitting the conductance curve to a dwave DOS. The superconducting gap features are considerably more broadened than found on optimal or overdoped Bi2212 as seen in Figs. 1 and 2. Also, the hump features are relatively more pronounced and for the most underdoped crystal the hump is at $\sim 200 \mathrm{meV}$, again slightly more than three times the superconducting gap, $\Delta=$ $65 \mathrm{meV}$. The location of the hump and its doping dependence is very similar to the hump found in the spectral weight function in ARPES near the $(\pi, 0)$ point [8]. From observation it appears that the superconducting gap feature is emerging out of a weak depression in the electronic density of states. The dashed lines of Fig. 3 represent 7 th order polynomial fits to the entire spectrum. These fits suggest a background shape that looks like a high energy pseudogap. Note that the minima of the fitted curves is not at the Fermi energy (zero bias). While this might seem unusual, such behavior is in fact found in spin-fermion FLEX calculations of a magnetic pseudogap [9]. In these models, the high energy pseudogap is due to short range antiferromagnetic order and is essentially a precursor of the SDW gap of the parent insulator. The dynamics prevents a fully developed gap to form and instead a pseudogap is found near $(\pi, 0)$.

\section{DISCUSSION AND SUMMARY}

The similarity of the peak/dip/hump features observed in tunneling to that found in ARPES [8] suggests that they are the same phenomena. Two recent theoretical explanations of the ARPES spectral weight $[10,11]$ along the $(\pi, 0)$ direction are based on the scattering of electrons by collective spin excitations. In these models a sharp quasiparticle peak exists at energy, $\Delta$, followed by a dip feature which is due to the scattering off a collective mode, $\Omega \sim \Delta$. This produces a dip at $\sim 2 \Delta$, however in the underdoped regime the feature should be less than $2 \Delta$ due to the decreasing energy of the collective mode. We have preliminary evidence from SIS junctions that this is observed [12]. At still higher energies there is a recovery of this strong coupling effect and the spectral weight exhibits a broad hump feature due to scattering off incoherent spin excitations. The characteristic energy of this hump feature is set by the superexchange energy, J. From tunneling density of states we find that $\Omega_{h u m p} \sim 3 \Delta \sim 2 \mathrm{~J}$ over the entire doping region [6]. The combination of the dip/hump features in these models gives the appearance in the density of states of a high energy pseudogap with a characteristic en- 
ergy set by $\mathbf{J}$. This is generally referred to as a magnetic pseudogap but which is distinguished from the low energy pseudogap associated with precursor superconductivity and therefore linked to the pairing gap. What is not clear at the moment is whether a magnetic pseudogap exists in the absence of pairing fluctuations as suggested in FLEX calculations [9]. Certainly the PES measurements in La214 indicate a magnetic pseudogap at temperatures much higher than $\mathrm{T}_{c}$ but its energy scale is still $\sim 3 \Delta$.

In conclusion we have found a connection between various experimental probes that suggest a high energy pseudogap scaling with $\mathbf{J}$ and the dip/hump features found in tunneling. Theoretical spin-fermion models seem to provide a straightforward explanation of the phenomena in terms of the scattering of electrons off collective spin excitations in the superconducting state. This magnetic pseudogap appears to be linked to the superconducting gap which might simply be an indication that the underlying interactions responsible for both gaps are the same.

\section{REFERENCES}

1. N. Miyakawa et al, Phys. Rev. Lett. 83 (1999) 1081 .

2. Tom Timusk and Bryan Statt, Rep. Prog. Phys. 62 (1999) 61.

3. M. Rubausen et al, Phys. Rev. B 58 (1998) 3462.

4. J. C. Irwin et al, in Studies of High Temperature Superconductors Vol. 27 (Nova Science, New York, 1999).

5. T. Sato et al, Phys. Rev. Lett. 83 (1999) 2254.

6. See N. Miyakawa et al, these proceedings.

7. Y. DeWilde et al, Phys. Rev. Lett. 80 (1998) 153.

8. J. C. Campuzano et al, Phys. Rev. Lett. 83 (1999) 3709.

9. J. Schmalian and K. H. Bennemann, in Studies of High Temperature Superconductors Vol. 27 (Nova Science, New York, 1999).

10. A. Abanov and A.V. Chubukov, Phys. Rev. Let.t. 83 (1999) 1652.

11. Jian-Xin Li et al, cond-mat/9912488.

12. J.F. Zasadzinski et al, (unpublished). 